\title{
Heterogeneidade de variâncias na avaliação genética de búfalas no Brasil
}

\section{Antonia Kécya França Moita ${ }^{1}$, Paulo Sávio Lopes ${ }^{2}$, Robledo de Almeida Torres ${ }^{2}$, Ricardo Frederico Euclydes ${ }^{2}$, Humberto Tonhati ${ }^{3}$, Ary Ferreira de Freitas ${ }^{4}$}

${ }^{1}$ Doutoranda em Genética e Melhoramento/UFV.

2 Departamento de Zootecnia/UFV.

${ }^{3}$ Departamento de Zootecnia/FCAV/UNESP, Jaboticabal, SP.

${ }^{4}$ Embrapa Gado de Leite.

RESUMO - Registros de produção de leite de 754 búfalas da raça Murrah foram utilizados com o objetivo de avaliar o efeito da heterogeneidade de variâncias na avaliação genética. Os componentes de covariância foram estimados pelo método da máxima verossimilhança restrita utilizando-se quatro modelos bicaracterísticos, considerando, como efeitos fixos, estação de parto e rebanho-ano de parto, e idade da vaca como covariável (efeito linear e quadrático). Os quatro modelos utilizados foram: modelo aditivo; modelo de repetibilidade; modelo aditivo com inclusão interação reprodutor $\times$ rebanho-ano; modelo de repetibilidade com inclusão da interação reprodutor $\times$ rebanho-ano. Os rebanhos foram classificados em duas classes de desviopadrão fenotípico para produção de leite e análises bicaracterísticas foram realizadas considerando cada classe de desvio-padrão como característica diferente. Foi conduzida também uma análise unicaracterística desconsiderando as classes de desvio-padrão fenotípico, incluindo o efeito da interação reprodutor $\times$ rebanho-ano. As estimativas de componentes de variância genética aditiva foram maiores na classe de alto desvio-padrão, comparadas às de baixo desvio-padrão. A maioria dos animais selecionados nos arquivos sem estratificação foi selecionada para alto desvio-padrão. Apesar do aumento nas variâncias aditivas e do erro nas de classes de alto desvio-padrão, suas herdabilidades foram menores, com exceção do modelo 2, cujo herdabilidade foi maior para a classe de alto desvio-padrão. Quando rebanhos são classificados em alto e baixo desvio-padrão fenotípico e a produção de leite nas diferentes classes é avaliada em modelo multicaracterística, a avaliação genética considera a heterogeneidade de variâncias entre rebanhos.

Palavras-chave: estratificação dos dados, melhoramento genético de búfalos

\section{Heterogeneity of variances on genetic evaluation of buffaloes in Brazil}

\begin{abstract}
Milk yield records of 754 Murrah female buffaloes were used to evaluate the effects of heterogeneity of variance among herds on genetic evaluation. The restricted maximum likelihood method was used to estimate the (co)variance components using four bi-trait models, considering season and herd-year of birth as fixed effects and age of the cow as covariable (linear and quadratic effects). The following models were used: additive; repeatability; additive with sire $\times$ herd-year interaction; and repeatability with sire $\times$ herd-year interaction. The herds were classified in two classes of phenotipic standard deviation for milk production and bi-traits analyses were carried out considering each class of standard deviation as a different characteristic. A single trait analysis was also carried out, disregarding phenotypic standard deviation classes, including sire $x$ herd-year interaction effect. The estimates of additive genetic variance components were higher in the high standard deviation class than those of low standard deviation. Most of the animals selected from files without stratification was selected for high standard deviation. Despite of the increase in additive variances and the error in high standard deviation classes, their heritability were lower, except for model 2, whose heritability was higher for the class with high standard deviation. When herds are classified into high and low phenotypic standard deviation and milk production in the different classes is evaluated in a model trait, genetic evaluation takes into account the heterogeneity of variances among herds.
\end{abstract}

Key Words: buffalo breeding, data stratification

\section{Introdução}

De acordo com Tonhati (1997), a quase totalidade dos criatórios de búfalos no Brasil adota regime de criação extensivo, caracterizado pela falta de controle zootécnico, sanitário e nutricional, desconhecendo o desempenho dos búfalos quanto ao ganho em peso, produção e qualidade do leite e características reprodutivas.

Estimativas de componentes de variâncias têm se mostrado heterogêneas de acordo com diferentes níveis de 
produção e classes de desvio-padrão fenotípico dos rebanhos (Araújo et al., 2001; Torres et al., 1999). Esta propriedade das variâncias proporciona desvios, acarretando riscos de serem selecionadas maiores proporções de animais dos rebanhos de maior variabilidade fenotípica e não necessariamente de maior valor genético, podendo, assim, reduzir o progresso genético esperado (Hill et al., 1983).

Gianola (1986) sugeriu uma análise multicaracterística, na qual os arquivos dos rebanhos com diferentes variâncias poderiam ser considerados como características diferentes. Entretanto, esse método requer estimativas de componentes de variância para cada rebanho.

Uma alternativa para solucionar os problemas associados à heterogeneidade de variâncias seria a estratificação dos dados baseada em algum critério, e posterior análise de características múltiplas (Gianola, 1986), tendo como princípio a proposta apresentada por Falconer (1952), de considerar a expressão de um genótipo em diferentes ambientes, como características distintas.

De acordo com Martins (2002), de forma geral, três situações de ocorrência de heterogeneidade de variância podem ser discriminadas. A primeira situação é aquela em que há apenas heterogeneidade de variância residual, indicando que a fonte de heterogeneidade não afeta as diferenças entre genótipos, porém altera a magnitude dos resíduos. A segunda é aquela em que há apenas heterogeneidade de variância genética, indicando que a fonte de heterogeneidade de variância afeta a magnitude das diferenças entre os genótipos, mas não afeta a magnitude dos resíduos. A terceira é aquela em que há heterogeneidade de variância genética e residual, indicando que a fonte de heterogeneidade afeta tanto a magnitude das diferenças entre genótipos como dos resíduos.

Com este estudo, objetivou-se avaliar o efeito da heterogeneidade de variâncias na avaliação genética de búfalas leiteiras no Brasil.

\section{Material e Métodos}

Os dados são provenientes de rebanhos bubalinos, coletados pelo Controle Leiteiro do Departamento de Zootecnia da Universidade Estadual de São Paulo - UNESP, câmpus de Jaboticabal.

Os dados foram editados de forma a eliminar registros de lactações incompletas e encerradas por causas anormais de secagem, lactações inferiores a 64 dias e registros após 305 dias de lactação. A idade das búfalas ao parto variou de 24 a 266 meses.
Os meses de parto das búfalas foram agrupados em duas épocas: época 1, de abril a setembro, que corresponde aos meses de menor incidência de chuvas; e época 2, de outubro a março, em que há maior precipitação pluviométrica, à exceção de um rebanho situado na região Nordeste, onde se considerou o inverso. Posteriormente, os efeitos fixos de rebanho e ano foram agrupados em subclasses, sendo exigidas, no mínimo, quatro observações por subclasse de rebanho-ano.

Feitas as edições necessárias, o arquivo de dados continha 1.774 lactações, oriundas de 754 búfalas da raça Murrah, filhas de 39 reprodutores, as quais pariram no período de 1987 a 2005, distribuídas em treze rebanhos, doze no Estado de São Paulo e um no Estado do Ceará. A duração da lactação foi de 64 a 305 dias e a idade ao parto variou de 24 a 185 meses. A conexidade dos dados foi feita para avaliar reprodutor x rebanho em função das filhas, para isso, foi exigido que cada reprodutor tivesse, no mínimo, duas filhas distribuídas em mais de um rebanho.

As estimativas dos componentes de variância e parâmetros genéticos e os valores genéticos preditos foram obtidos utilizando o programa MTDFREML (Multiple Trait Derivative Free Restricted Maximum Likelihood), descrito por Boldman et al. (1995), utilizando modelo animal. Esse programa utiliza o algoritmo simplex para localizar o mínimo de - $2 \log _{\mathrm{e}} \mathrm{L}$, sendo $\mathrm{L}$ a função de verossimilhança; os componentes de covariância que minimizam a função $-2 \log _{\mathrm{e}} \mathrm{L}$ são estimativas de verossimilhança. Utilizou-se como critério de convergência a variância dos valores simples, igual a $10^{-9}$. Para conferir a convergência repetiu-se a análise e observou-se o valor do -2 $\log _{\mathrm{e}} \mathrm{L}$, de modo a observar se os valores foram iguais até a quarta casa decimal.

Com o intuito de investigar a heterogeneidade de variâncias, utilizou-se o desvio-padrão fenotípico dos rebanhos para produção de leite até 305 dias de lactação, como critério para estratificar os dados em duas classes, com número aproximadamente igual de observações. Os rebanhos foram classificados em duas classes de desviopadrão fenotípico para produção de leite e efetivaram-se análises bicaracterísticas, considerando-se cada classe de desvio-padrão como característica diferente. Foi conduzida, também, uma análise unicaracterística, na qual foram desconsideradas as classes de desvio-padrão fenotípico, incluindo o efeito da interação reprodutor x rebanho-ano. A classe de baixo desvio-padrão fenotípico corresponde aos dados cujo desvio-padrão da produção de leite do rebanho foi menor ou igual a $600 \mathrm{~kg}$, e a de alto desvio-padrão fenotípico, maior que $600 \mathrm{~kg}$.

$\mathrm{O}$ arquivo de pedigree que originou a matriz de numeradores dos coeficientes de parentesco (NRM), 
utilizada em todas as análises, continha 864 animais diferentes, 1.776 elementos não-zero na NRM e nenhum animal endogâmico, consequentemente, o coeficiente médio de endogamia foi igual a zero.

As análises foram conduzidas utilizando-se quatro modelos bcaracterísticos, nos quais foram considerados como efeitos fixos estação de parto e rebanho-ano de parto e idade da vaca como covariável (efeito linear e quadrático). Os efeitos aleatórios dos quatro modelos são descritos a seguir: modelo bicaracterístico aditivo (efeito aditivo e erro); modelo bicaracterístico de repetibilidade (modelo 1 mais efeito de ambiente permanente); modelo bicaracterístico aditivo com a interação reprodutor $\times$ rebanho-ano (modelo 1 incluindo a interação reprodutor $\times$ rebanho-ano). Modelo bicaracterístico de repetibilidade com a interação reprodutor $\times$ rebanho-ano (modelo 2 incluindo a interação reprodutor $\times$ rebanho-ano).

A estimativa da estatística do teste da razão de verossimilhança (LR) foi comparada com o valor obtido por meio da distribuição de qui-quadrado, com 1 grau de liberdade. A estimativa foi obtida pela expressão:

$$
\begin{aligned}
& \mathrm{LR}_{\mathrm{ij}}=-2 \log _{\mathrm{e}}\left(\mathrm{L}_{\mathrm{j}} / \mathrm{L}_{\mathrm{i}}\right), \\
& \mathrm{LR}_{\mathrm{ij}}=2 \log _{\mathrm{e}}\left(\mathrm{L}_{\mathrm{i}}\right)-2 \log _{\mathrm{e}}\left(\mathrm{L}_{\mathrm{j}}\right), \\
& \mathrm{LR}_{\mathrm{ij}}=2\left[\log _{\mathrm{e}}\left(\mathrm{L}_{\mathrm{i}}\right)-\log _{\mathrm{e}}\left(\mathrm{L}_{\mathrm{j}}\right)\right],
\end{aligned}
$$

$\mathrm{Na}$ qual $\mathrm{LR}_{\mathrm{ij}}=$ estatística do teste da razão de verossimilhança para modelos sequencialmente reduzidos; $\mathrm{L}_{\mathrm{i}}=$ máximo da verossimilhança para o modelo completo $\mathrm{i}$; e $\mathrm{L}_{\mathrm{j}}=$ máximo da verossimilhança para o modelo reduzido $\mathrm{j}$.

A hipótese de nulidade a ser testada implicou que as funções de verossimilhança dos modelos completo e reduzido não diferiam entre si, ou seja,

$$
H_{0}:-2 \log _{e}\left(L_{i}\right)=-2 \log _{e}\left(L_{j}\right) \text {, }
$$

A regra decisória empregada foi: $\mathrm{se}_{\mathrm{LR}}>\chi_{\mathrm{ij}}^{2}$, o teste era significativo e o modelo completo fornecia maior valor da função de verossimilhança em relação ao modelo reduzido; se $\mathrm{LR}_{\mathrm{ij}}<\chi_{\text {tab }}^{2}$, o teste era não-significativo e o modelo completo não fornecia maior valor da função de verossimilhança em relação ao modelo reduzido.

Os valores genéticos aditivos preditos dos reprodutores para produção de leite até 305 dias de lactação, incluindo ou não no modelo o termo da interação reprodutor $\times$ rebanhoano, foram organizados em arquivos, objetivando verificar possíveis alterações no ordenamento das predições dos valores genéticos aditivos dos reprodutores, quando o termo da interação reprodutor $\times$ rebanho-ano foi incluído no modelo por meio da obtenção da correlação de Spearman e de Pearson.

\section{Resultados e Discussão}

Os rebanhos que foram classificados como de baixo desvio-padrão apresentaram baixa produção, com exceção do rebanho 1011, que possui baixo desvio-padrão e alta produção, assim como os que apresentaram alto desviopadrão apresentaram alta produção.

Araújo (2000) e Torres et al. (1999) trabalharam com bovinos da raça Holandesa e também observaram que as médias de produção de leite do rebanho aumentaram da classe de baixo desvio-padrão fenotípico para a classe de alto desvio-padrão fenotípico do rebanho.

As inclusões do efeito de ambiente permanente (modelo 2 vs modelo 1 ), da interação reprodutor $\times$ rebanho-ano no modelo de aditivo (modelo 3 vs 1 ) e da interação reprodutor $\times$ rebanho-ano no modelo de repetibilidade (modelo 4 vs modelo 2) não foram significativas.

As estimativas de componentes de variância genética aditiva para produção de leite foram maiores na classe de alto desvio-padrão, comparadas às de baixo desvio-padrão. Entre os modelos, a maior variância aditiva foi verificada no modelo 1, para a classe de alto desvio-padrão. Enquanto as estimativas de componentes de variâncias residuais foram maiores no modelo 2, para a classe de alto desvio-padrão.

Torres et al. (1999), Costa (1999) e Short et al. (1990), em pesquisa com vacas da raça Holandesa, também observaram maiores valores nas estimativas de componentes de variância genética aditiva e residual nas classes de maior desvio-padrão fenotípico. Ao passo que Boldman \& Freeman (1990) obtiveram os mesmos resultados trabalhando com médias de produção em vacas.

Houve diminuição na estimativa do componente de variância do efeito de ambiente permanente quando o efeito da interação reprodutor $\times$ rebanho-ano foi acrescentado.

Tabela 1 - Rebanhos, número de dados e classe de desvios-padrão nos rebanhos de búfalas da raça Murrah

\begin{tabular}{lcccc}
\hline Rebanhos & Média & EPM & Classe & Dados \\
\hline 1008 & 1056,020 & 225,4957 & 1 & 5 \\
1005 & 942,117 & 250,14 & 1 & 30 \\
1037 & 1450,873 & 252,43 & 1 & 12 \\
1038 & 1335,806 & 263,28 & 1 & 7 \\
1006 & 1031,315 & 356,24 & 1 & 84 \\
1004 & 1109,669 & 433,73 & 1 & 29 \\
1054 & 1484,081 & 488,61 & 1 & 47 \\
1011 & 1805,115 & 514,77 & 1 & 567 \\
1001 & 1350,294 & 526,69 & 1 & 91 \\
1002 & 1663,620 & 601,99 & 2 & 104 \\
1010 & 1681,672 & 687,003 & 2 & 544 \\
1012 & 2633,542 & 763,26 & 2 & 171 \\
1032 & 1776,071 & 788,03 & 2 & 83 \\
Média geral & 1736,663 & 705,85 & - & - \\
\hline
\end{tabular}

R. Bras. Zootec., v.39, n.7, p.1443-1449, 2010 
Tabela 2 - Valores de -2logaritmo natural da função de verossimilhança para os modelos e classes consideradas

\begin{tabular}{lccc}
\hline Modelo & $-2 \log _{\mathrm{e}} \mathrm{L}$ & $\mathrm{LR}$ & Valor de $\mathrm{p}$ \\
\hline M1 & $22.589,8071$ & - & - \\
M2 & $22.584,7722$ & 5,034958 & 0,10252897 \\
M3 & $22.583,7781$ & 6,029042 & 0,146282651 \\
M4 & $22.586,4911$ & 1,718899 & 0,143424328 \\
\hline
\end{tabular}

M1 = modelo bicaracterístico aditivo; M2 = modelo bicaracterístico que considera o efeito de ambiente permanente; M3 = modelo bicaracterístico que considera o efeito da interação reprodutor $\times$ rebanho-ano; e M4 = modelo bicaracterístico que considera os efeitos de ambiente permanente e da interação reprodutor $\times$ rebanho-ano.

A inclusão do efeito de ambiente permanente no modelo diminuiu o valor da estimativa de herdabilidade de 0,50 para 0,13 na classe de baixo desvio-padrão, e de 0,44 para 0,40 nas classes de alto desvio-padrão nos modelos 1 e 2 . A inclusão da interação reprodutor $\times$ rebanho-ano no modelo aditivo não modificou os valores estimados para herdabilidade nas classes de baixo desvio-padrão e reduziu de 0,44 para 0,43 nas classes de alto desvio-padrão. Entretanto, a inclusão da interação reprodutor $\times$ rebanho-ano no modelo de repetibilidade, causou aumento na estimativa de herdabilidade de 0,40 para 0,41 na classe de alto desvio-padrão e aumento de 0,13 para 0,49 na classe de baixo desvio-padrão fenotípico.

Com exceção do modelo 2, as estimativas de herdabilidade nas classes de baixo desvios-padrão apresentaram-se maiores que nas de alto desvio-padrão. Entretanto, Torres et al. (1999), Short et al. (1990) e Dong \& Mao (1990) verificaram maiores estimativas de herdabilidade para produção de leite em bovinos da raça Holandesa nas classes de alto desviopadrão fenotípico. Mesmo resultado foi obtido por Hill et al. (1983), trabalhando com médias de produção.

A menor amplitude entre os valores genéticos preditos para os animais ocorreu na classe de baixo desvio-padrão do modelo 2, e a maior na de alto desvio-padrão do modelo 1 (Tabela 5). Todavia, não houve grandes diferenças entre os quatro modelos. O modelo 4 apresentou os menores valores para média (alto desvio-padrão), ao passo que o modelo 2 apresentou o menor valor de desvio-padrão (baixo desviopadrão).

As correlações entre as classes de baixo e alto desviopadrão fenotípico foram de 0,92; 0,99, 0,94 e 0,89; respectivamente para os modelos M1, M2, M3 e M4.

Tabela 3 - Estimativas dos componentes de variância para produção de leite em búfalas, nas classes de desvios-padrão fenotípico alto e baixo

\begin{tabular}{|c|c|c|c|c|c|}
\hline \multirow[t]{2}{*}{ Modelo } & \multicolumn{5}{|c|}{ Componente de variância } \\
\hline & Desvio-padrão & $\sigma_{\mathrm{a}}^{2}$ & $\sigma_{\mathrm{c}^{3}}^{2}$ & $\sigma_{\mathrm{c}^{1}}^{2}$ & $\sigma_{\mathrm{e}}^{2}$ \\
\hline M1 & Baixo & $112.276,41546$ & - & - & $113.693,10166$ \\
\hline \multirow[t]{2}{*}{ M2 } & Baixo & $27.740,07416$ & $70.945,9$ & - & $111.856,04974$ \\
\hline & Alto & $144.399,85993$ & $10.048,2$ & - & $207.697,35301$ \\
\hline M3 & Baixo & $113.376,35133$ & - & 292,209 & $113.404,29226$ \\
\hline M4 & Alto & $143.969,47494$ & 661,815 & 9170,65 & $199.846,96386$ \\
\hline
\end{tabular}

M1 = modelo bicaracterístico aditivo, M2 = modelo bicaracterístico que considera o efeito de ambiente permanente, M3 = modelo bicaracterístico que considera o efeito da interação reprodutor $\times$ rebanho-ano; e M4 = modelo bicaracterístico que considera os efeitos de ambiente permanente e da interação reprodutor $\times$ rebanhoano; $\sigma_{a}^{2}=$ variância genética aditiva; $\sigma_{c^{1}}^{2}=$ variância de ambiente permanente; $\sigma_{c^{3}}^{2}=$ variância da interação reprodutor $\times$ rebanho-ano e $\sigma_{e}^{2}=$ variância residual.

Tabela 4 - Estimativas de herdabilidade, de repetibilidade e das proporções da variância fenotípica das classes de desvios-padrão fenotípico alto e baixo para produção de leite

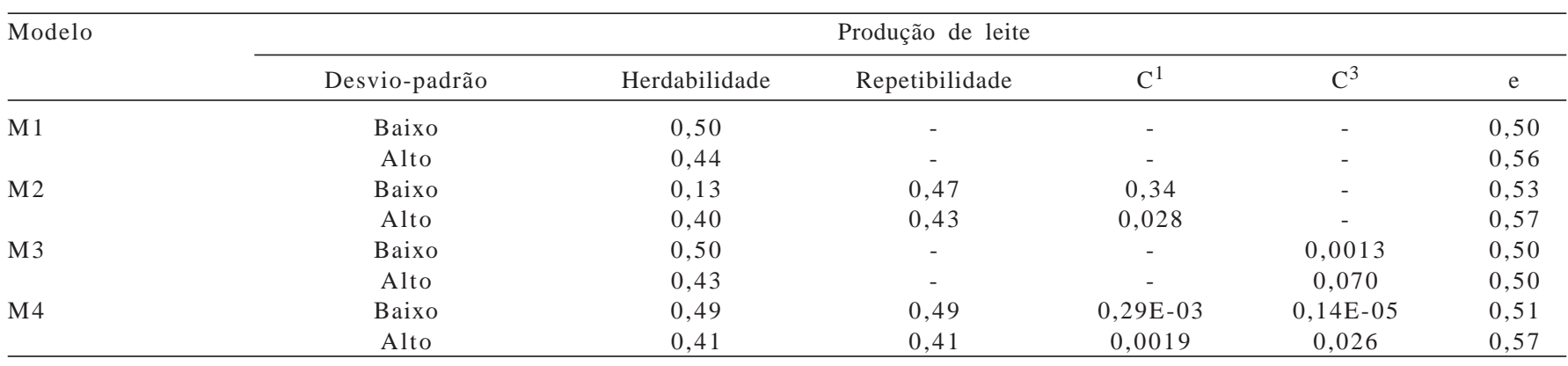

M1 = modelo bicaracterístico aditivo, M2 = modelo bicaracterístico que considera o efeito de ambiente permanente, M3 = modelo bicaracterístico que considera o efeito da interação reprodutor $\times$ rebanho-ano, e M4 = modelo bicaracterístico que considera os efeitos de ambiente permanente e da interação reprodutor $\times$ rebanho-ano; $\mathrm{C}^{1}=$ proporções da variância fenotípica atribuída aos efeitos de ambiente permanente, $\mathrm{C}^{3}=$ efeito da interação reprodutor $\times$ rebanho-ano, e $=$ efeito residual. 
Tabela 5 - Médias dos valores genéticos preditos para os animais, desvios-padrão, valores máximos, valores mínimos e amplitudes para as classes consideradas

\begin{tabular}{|c|c|c|c|c|c|}
\hline \multicolumn{2}{|c|}{ Modelo } & \multicolumn{4}{|c|}{ Valor genético predito } \\
\hline & & Média & Desvio-padrão & Mínimo & Máximo \\
\hline M1 & Baixo & 0,7473 & 205,6501 & $-626,9191$ & 1000 \\
\hline \multirow[t]{2}{*}{ M2 } & Baixo & $-2,4104$ & 95,8084 & $-321,3162$ & 443,2239 \\
\hline & Alto & $-8,0392$ & 227,1585 & $-769,3084$ & 1069 \\
\hline M3 & Baixo & 1,3008 & 208,6743 & $-629,6201$ & 1004 \\
\hline M4 & Alto & $-8,2364$ & 241,2110 & $-794,7596$ & 1137 \\
\hline
\end{tabular}

M1 = modelo bicaracterístico aditivo, M2 = modelo bicaracterístico que considera o efeito de ambiente permanente, M3 = modelo bicaracterístico que considera o efeito da interação reprodutor $\times$ rebanho-ano, e M4 = modelo bicaracterístico que considera os efeitos de ambiente permanente e da interação reprodutor $\times$ rebanho-ano.

As correlações de Pearson e Spearman obtidas entre os dados do arquivo sem estratificação (análise geral) e os dados das classes de alto desvio-padrão foram maiores em todos os modelos quando comparados com os das classes de baixo desvio-padrão. Isso indica que os animas selecionados nos arquivos sem estratificação provavelmente seriam, na maioria, da classe de alto desvio-padrão (Tabela 6).

As menores correlações de Pearson e Spearman foram observadas no modelo 4, entre o arquivo sem estratificação e a classe de baixo desvio-padrão, e entre as classes de baixo e alto desvio-padrão fenotípico. Resultados diferentes foram obtidos por Costa (1999), que encontrou correlações genéticas próximas à unidade para produção de leite em bovinos da raça Holandesa entre as classes de alto e baixo desvio-padrão.

De acordo com Martins (2002), quando as correlações genéticas entre as classes da fonte de heterogeneidade de variância não forem iguais à unidade, conforme verificado no presente trabalho, uma consequência adicional ocorreria porque a avaliação genética tenderia a indicar como superiores os genótipos cuja capacidade adaptativa fosse mais estável, o que não significa, obrigatoriamente, que tais genótipos sejam superiores em todos os ambientes.

Houve grande alteração no ordenamento dos animais com base nos valores genéticos preditos, quando se utilizaram as classes de baixo desvio-padrão nos quatro modelos estudados. Nos 20 melhores animais, 45\% nas classes de baixo desvio-padrão e que $80 \%$ foram comuns nas classes alto desvio-padrão (Tabela 7) e variou de 0,45 a 0,90 quando se compararam baixo e alto desvios-padrão fenotípico dos modelos 2 e 3 , respectivamente.

Ao comparar os 20 melhores animais do modelo com a interação reprodutor $\times$ rebanho-ano, sem estratificação ao modelo 1 , observa-se que $60 \%$ deles são comuns aos de baixo desvio-padrão e $85 \%$ deles são comuns aos de alto desvio-padrão (Tabela 8). Esses resultados confirmam os valores obtidos nas correlações de Pearson e de Spearman (Tabela 6).

Tabela 6 - Coeficiente de correlação de Pearson e de Spearman entre os valores genéticos preditos para produção de leite

\begin{tabular}{|c|c|c|c|c|c|c|c|c|}
\hline \multirow{3}{*}{ Modelo } & \multicolumn{8}{|c|}{ Correlação de Pearson } \\
\hline & \multicolumn{2}{|c|}{ M1 } & \multicolumn{2}{|c|}{ M2 } & \multicolumn{2}{|c|}{ M3 } & \multicolumn{2}{|c|}{ M4 } \\
\hline & BDP & ADP & $\mathrm{BDP}$ & ADP & BDP & ADP & BDP & $\mathrm{ADP}$ \\
\hline $\begin{array}{l}\text { Geral } \\
\text { BDP }\end{array}$ & 0,9405 & $\begin{array}{l}0,9894 \\
0,9214\end{array}$ & 0,9784 & $\begin{array}{l}0,9749 \\
0,9965\end{array}$ & 0,9463 & $\begin{array}{l}0,9922 \\
0,9398\end{array}$ & 0,8517 & $\begin{array}{l}0,9662 \\
0,8862\end{array}$ \\
\hline Modelo & M1 & \multicolumn{5}{|c|}{ Correlação de Spearman } & M4 & \\
\hline $\begin{array}{l}\text { Geral } \\
\text { BDP }\end{array}$ & $\begin{array}{c}\text { BDP } \\
0,9626\end{array}$ & $\begin{array}{c}\text { ADP } \\
0,9877 \\
0,9405\end{array}$ & $\begin{array}{c}\text { BDP } \\
0,9792\end{array}$ & $\begin{array}{c}\text { ADP } \\
0,9771 \\
0,9970\end{array}$ & $\begin{array}{c}\text { BDP } \\
0,9649\end{array}$ & $\begin{array}{c}\text { ADP } \\
0,9906 \\
0,9541\end{array}$ & $\begin{array}{c}\text { BDP } \\
0,8790\end{array}$ & $\begin{array}{c}\text { ADP } \\
0,9632 \\
0,9167\end{array}$ \\
\hline
\end{tabular}

M1 = modelo bicaracterístico aditivo, M2 = modelo bicaracterístico que considera o efeito de ambiente permanente, M3 = modelo bicaracterístico que considera o efeito da interação reprodutor $\times$ rebanho-ano, e M4 = modelo bicaracterístico que considera os efeitos de ambiente permanente e da interação reprodutor $\times$ rebanhoano; modelo geral = considera os efeitos de ambiente permanente e da interação reprodutor $\times$ rebanho-ano sem que haja a separação em baixo e alto desvio-padrão; BDP = baixo desvio-padrão, ADP = alto desvio-padrão. 
Tabela 7 - Valores genéticos dos vinte melhores animais nos modelos M1, M2, M3 e M4

\begin{tabular}{|c|c|c|c|c|c|c|c|c|c|c|c|c|c|c|c|}
\hline \multicolumn{4}{|c|}{ Modelo 1} & \multicolumn{4}{|c|}{ Modelo 2} & \multicolumn{4}{|c|}{ Modelo 3} & \multicolumn{4}{|c|}{ Modelo 4} \\
\hline IA & BDP & IA & ADP & IA & BDP & IA & ADP & IA & BDP & IA & ADP & IA & BDP & IA & ADP \\
\hline 535 & 1000,379 & 145 & 1168,944 & 145 & 443,224 & 145 & 1068,893 & 535 & 991,116 & 145 & 1137,354 & 535 & 1004,493 & 145 & 1183,445 \\
\hline 763 & 854,444 & 417 & 1077,823 & 417 & 403,830 & 417 & 1013,596 & 763 & 843,524 & 417 & 1035,894 & 763 & 858,005 & 417 & 1048,608 \\
\hline 145 & 790,902 & 535 & 951,610 & 648 & 353,962 & 648 & 862,032 & 145 & 742,122 & 648 & 891,726 & 145 & 826,990 & 535 & 980,187 \\
\hline 494 & 680,391 & 648 & 926,125 & 158 & 336,172 & 830 & 821,172 & 494 & 683,855 & 535 & 838,190 & 494 & 675,775 & 648 & 942,754 \\
\hline 708 & 640,527 & 830 & 863,203 & 830 & 324,581 & 158 & 783,019 & 708 & 620,164 & 158 & 831,005 & 158 & 667,215 & 158 & 868,506 \\
\hline 158 & 640,304 & 158 & 850,996 & 633 & 302,435 & 633 & 741,661 & 158 & 609,452 & 830 & 822,429 & 708 & 643,662 & 830 & 828,209 \\
\hline 648 & 577,676 & 633 & 783,306 & 707 & 288,661 & 780 & 697,722 & 231 & 544,719 & 633 & 747,572 & 648 & 616,059 & 707 & 782,564 \\
\hline 417 & 550,148 & 707 & 741,468 & 780 & 273,739 & 707 & 690,776 & 495 & 527,473 & 707 & 729,545 & 417 & 576,777 & 763 & 761,791 \\
\hline 231 & 545,744 & 708 & 737,029 & 778 & 267,203 & 778 & 681,851 & 648 & 526,093 & 780 & 701,529 & 231 & 546,231 & 633 & 761,626 \\
\hline 495 & 531,123 & 780 & 727,820 & 535 & 267,106 & 88 & 612,312 & 546 & 517,634 & 778 & 667,810 & 495 & 532,945 & 708 & 732,683 \\
\hline 546 & 527,893 & 763 & 720,881 & 533 & 255,386 & 533 & 606,225 & 563 & 511,945 & 708 & 666,036 & 546 & 528,760 & 780 & 720,628 \\
\hline 563 & 519,272 & 778 & 708,222 & 156 & 248,921 & 156 & 605,156 & 92 & 503,022 & 372 & 653,436 & 707 & 521,879 & 372 & 700,401 \\
\hline 92 & 517,746 & 372 & 678,952 & 638 & 245,760 & 372 & 596,223 & 456 & 499,921 & 156 & 645,274 & 563 & 521,364 & 156 & 683,469 \\
\hline 694 & 504,645 & 156 & 660,832 & 372 & 241,073 & 638 & 590,569 & 694 & 497,176 & 763 & 626,571 & 92 & 513,309 & 778 & 665,125 \\
\hline 456 & 503,414 & 533 & 655,573 & 599 & 232,359 & 829 & 579,141 & 417 & 485,015 & 533 & 621,854 & 694 & 507,867 & 599 & 658,519 \\
\hline 533 & 499,652 & 638 & 638,725 & 708 & 231,279 & 535 & 574,734 & 533 & 471,097 & 599 & 591,808 & 456 & 507,482 & 533 & 638,262 \\
\hline 707 & 476,513 & 599 & 600,822 & 88 & 224,949 & 781 & 572,910 & 540 & 465,671 & 88 & 581,095 & 533 & 500,198 & 135 & 607,088 \\
\hline 540 & 469,664 & 829 & 596,964 & 829 & 224,902 & 498 & 559,690 & 707 & 454,742 & 638 & 577,973 & 599 & 489,742 & 412 & 591,993 \\
\hline 633 & 452,639 & 88 & 592,100 & 781 & 222,336 & 599 & 546,123 & 454 & 442,162 & 781 & 577,196 & 372 & 473,813 & 638 & 582,772 \\
\hline 372 & 446,695 & 781 & 589,189 & 763 & 219,646 & 698 & 535,413 & 550 & 429,667 & 829 & 565,279 & 540 & 470,489 & 781 & 581,050 \\
\hline
\end{tabular}

IA = identificação do animal; M1 = modelo bicaracterístico aditivo, M2 = modelo bicaracterístico que considera o efeito de ambiente permanente, M3 = modelo bicaracterístico que considera o efeito da interação reprodutor $\times$ rebanho-ano, e M4 = modelo bicaracterístico que considera os efeitos de ambiente permanente e da interação reprodutor $\times$ rebanho-ano; BDP = baixo desvio-padrão, ADP = alto desvio-padrão.

Tabela 8 - Valores genéticos das 20 melhores búfalas em análise de característica simples (modelo geral) e nas classes de desvio-padrão fenotípico

\begin{tabular}{|c|c|c|c|c|c|}
\hline \multicolumn{2}{|c|}{ Modelo geral } & \multicolumn{4}{|c|}{ Modelo 1} \\
\hline 145 & 1196,1992 & 535 & 1000,379 & 145 & 1168,944 \\
\hline 648 & 932,1694 & 145 & 790,902 & 535 & 951,610 \\
\hline 535 & 916,5317 & 494 & 680,391 & 648 & 926,125 \\
\hline 158 & 866,8978 & 708 & 640,527 & 830 & 863,203 \\
\hline 633 & 789,3455 & 417 & 550,148 & 707 & 741,468 \\
\hline 707 & 729,1452 & 231 & 545,744 & 708 & 737,029 \\
\hline 372 & 704,5528 & 495 & 531,123 & 780 & 727,820 \\
\hline 780 & 692,3846 & 546 & 527,893 & 763 & 720,881 \\
\hline 533 & 681,6568 & 563 & 519,272 & 778 & 708,222 \\
\hline 778 & 670,212 & 92 & 517,746 & 372 & 678,952 \\
\hline 135 & 615,8406 & 540 & 469,664 & 829 & 596,964 \\
\hline 494 & 594,1671 & 633 & 452,639 & 88 & 592,100 \\
\hline 276 & 589,1460 & 372 & 446,695 & 781 & 589,189 \\
\hline
\end{tabular}

VG = valor genético; M1 = modelo bicaracterístico aditivo; e modelo geral = considera os efeitos de ambiente permanente e da interação reprodutor $\times$ rebanho-ano sem que haja a separação em baixo e alto desvio-padrão.

\section{Conclusões}

Ao selecionar animais sem considerar a heterogeneidade de variância, há tendência em favorecer aqueles pertencentes a rebanhos de alta variância, em detrimento aos de menor variabilidade fenotípica. A estratificação dos rebanhos de búfalos para a característica produção de leite em classes de alto e baixo desvio-padrão fenotípico corrige para a heterogeneidade de variância. Quando realizada a estratificação do rebanho pelo nível de produção, é recomendável na avaliação genética o uso de um modelo mais simples (apenas efeito aditivo). Caso não 
seja feita a estratificação dos rebanhos, é necessário utilizar um modelo mais completo (efeitos aditivo, permanente e da interação reprodutor $\times$ rebanho-ano).

\section{Agradecimentos}

Aos criadores de búfalos, pela perseverança na dedicação ao melhoramento da espécie. À Associação Brasileira de Criadores de Búfalos. Ao professor Humberto Tonhati, pela cessão dos dados sobre búfalos. Ao Conselho Nacional de Desenvolvimento Científico e Tecnológico (CNPq) e à Universidade Federal de Viçosa, pela oportunidade de estudar, aprender e desenvolver esta pesquisa.

\section{Referências}

ARAÚJO, C.V.; TORRES, R.A.; COSTA, C.N. Et Al. Efeito da interação reprodutor $\mathrm{x}$ rebanho na acurácia da avaliação genética de reprodutores da raça holandesa no Brasil. Revista Brasileira de Zootecnia, v.30, n.3, p.1000-1006, 2001 (supl. 1).

BOLDMAN, K.G.; KRIESE, L.A.; VAN VLECK, L.D. et al. A manual for use of MTDFREML: a set of programs to obtain estimates of variances and covariances (DRAFT). Lincoln: Department of Agriculture/Agriculture Research Service, 1995. 86p.

BOLDMAN, K.G.; FREEMAN, A.E. Adjustment for heterogeneity of variance by herd production level in dairy cow and sire evaluation. Journal of Dairy Science, v.73, n.2, p.503-512, 1990.

COSTA, C.N. Interação genótipo $\mathrm{x}$ ambiente em gado de leite. In: SIMPÓSIO INTERNACIONAL DE GENÉTICA E MELHORAMENTO ANIMAL, 1., 1999, Viçosa, MG. Anais... Viçosa, MG: UFV, 1999. p.161-191.

DONG, M.C.; MAO, I.L. Heterogeneity of (co)variance and heritability in different levels of intraherd milk production variance and of herd average. Journal of Dairy Science, v.73, p.843-851, 1990.

FALCONER, D.S. The problem of environment and selection. American Nature, v.86, n.830, p.293-298, 1952.

GIANOLA, D. Selection criteria and estimation of parameters when the variance is heterogeneous. Theoretical Applied Genetic, v.72, n.5, p.671-677, 1986.

HILL, W.G.; EDWARDS, M.R.; AHMED, M.K. A. et al. Heritability of milk yield and composition at different levels and variability of production. Animal Production, v.36, n.1, p.59-68, 1983.

MARTINS, E.N. Avaliação genética e heterogeneidade de variância. In: REUNIÃO ANUAL DA SOCIEDADE BRASILEIRA DE ZOOTECNIA, 34., 2002, Recife. Anais... Recife: Sociedade Brasileira de Zootecnia, 2002. p.205-214.

SHORT, T.H.; BLAKE, R.W.; QUAAS, R.L. et al. Heterogeneous within-herd variance: 1 - Genetic parameters for first and second lactation milk yield of grade Holstein cows. Journal of Dairy Science, v.73, n.11, p.3312-3320, 1990.

TONHATI, H. Melhoramento genético de bubalinos para carne e leite. In: OLIVEIRA, G.J.C; ALMEIDA, A.M.L.; SOUZA FILHO, U.A.S. (Eds.) O búfalo no Brasil. Cruz das almas: UFBA - Escola de Agronomia, 1997. p.101-113.

TORRES, R.A.; BERGMANN, J.A.G.; COSTA, C.N. et al. Ajustamento de variâncias para a produção de leite entre rebanhos da raça holandesa no Brasil. Revista Brasileira de Zootecnia, v.28, n.2, p.295-303, 1999. 